\title{
Analysis of Signal Propagation in Vehicular Communications with RFID Tags
}

\author{
Aaron Don M. Africa, John Arvin Mercado, Joshua Kenichi Sim \\ Department of Electronics and Communications Engineering \\ De La Salle University, Manila \\ 2401 Taft Ave., Malate, Manila 1004, \\ Philippines, aaron.africa@dlsu.edu.ph
}

\begin{abstract}
Radio-frequency identification (RFID) is a commonly used technology used in various applications such as inventory readers, body counters, marathon race tagging, and the like. The research to be performed in this paper aims to study how RFID systems can help ease traffic through the monitoring of vehicles and possibly be implemented into more advanced systems to deliver information to the masses for further use of remote monitoring and traffic management. Different sizes of RFID tags or antennas are to be tested and used to determine the efficiency of signal propagation in vehicles once the tag enters the RFID reader's range. Effectiveness will be measured by picking up signals from vehicles at the minimum radius for a lower cost. The environment will be simulated via MATLAB Simulink. The system will consist of a reader and a tag, where the reader serves as the detector, while the tag serves as the object of detection. In the simulated environment, the system will also take note of the different noises encountered in the channel which is present in real life such as multipath fading.
\end{abstract}

Key words: Radio-frequency Identification, RFID tag, RFID reader, traffic management, signal propagation, simulated environment, vector network analyzer, error detection, signal-to-noise ratio.

\section{INTRODUCTION}

Technology has been the driver of innovation and progress in most countries for the longest time. Ever since the two World Wars, technology has pushed mankind to a new era, the digital era. The need for technology in warfare has led to its numerous applications in times of peace. As people struggled for connectivity in the past, that problem is no longer an issue. In the world today, people are more connected than ever before. Not only people but several systems have integrated itself into the daily lives of the average person making their lives more convenient. The interconnectivity of things and processes was made possible by advancements in technology such as the RFID.

Radio Frequency Identification or also known as RFID, has been a technology in the works for the longest time. One of its earliest iterations was used in World War I when planes of the German armies would do a roll to signal the radar below that they were a friendly fighter jet. This was the first-time passive backscatter was utilized in a real-life application [1]. Nowadays however these systems have developed into something way more advanced and complex. Before the development of the RFID, systems used to make do with barcodes. The problem with systems such as this is the reliance of line of sight detection, this means any obstruction, would lead to something being undetectable [2]. This led to researchers developing new technology that did not rely online of sight transmission. This led to the development of the Radio Frequency Identification.

The RFID system mainly comprises of two major elements, the tag and the reader [3]. The tag of an RFID is usually a low-cost transponder that is utilized to be detectable by the reader [4], while the reader is the one that detects and sends signals to the tag [5]. These components serve as antennas, meaning things like size, cost, polarization, bandwidth, and the gain would affect its operationality [6,7]. The system can be utilized in numerous ways and is already being utilized in the production industry quite a lot. The focus of this paper however lies in the cars and traffic of the Philippines, which would mean the introduction of real-life noise such as multipath fading [8]. The integration of an RFID system could help not only track traffic but also ease the traffic by proper routing and traffic management [9].

\section{BACKGROUND OF THE STUDY}

Ever since the culmination of communication systems, the ability to send and receive signals has been constantly developing. Just like any other advancement in technology the main driving force was conflict and competition. During World War I the planes of a specific nation needed some manner of identification to indicate whether it is a friendly or a plane of the opposing army. The Germans thought of a way to disrupt radars, by letting their fighters do a roll in the air. This small action led the signals to be disrupted which indicated to ground troops that the planes were friendly, this was the first example of what is known as passive backscatter. Ever since that simple need, the communications industry has made several advancements in research and technology. 
Nowadays the ability to send and receive signals has become so commonplace that an average person can get their hands on one as it is available in the commercial market. Day by day new advancements is being made in this field of science. RFIDs have been developing constantly especially with the connection to the internet of things since it can be critical when it comes to interconnecting humans with the technology around them. Several industries make use of RFID technology, especially in the production and distribution line of work as they need to be able to track and monitor their products [10]. Lately, the technology has reached a point where sensitivity and displacement can be detected up to the nanometers, and sensors have developed to a point where it has a chipless RFID tag [11]. Not only that but with the invention of $3 \mathrm{D}$ printing it is now even possible to $3 \mathrm{D}$ print composites with an embedded microchip that serves as an RFID [12]. With technology aiming for integration and convenience as it connects humans to the tools around them, the applications of the RFID seem endless and is booming with potential.

\section{STATEMENT OF THE PROBLEM}

As time passes by, and as the world progresses with technology the automobile industry is booming now more than ever. This specific increase in automobiles calls for bigger roads and better infrastructure, something that is not seen in most developing countries. This causes traffic. Traffic is a significant problem that hinders progress in any part of the world. Especially in large urban areas, traffic congestion and tidal flow management is a growing problem because of the increase in the number of vehicles in all countries. Some of the traffic concerns are congestion and accidents which have been known to cause noise pollution, long travel times, property damage, increased fuel usages, and heavy environmental pollution [13]. In urban areas, traffic signal controllers play an important role to improve the efficiency of vehicles, traffic congestion and hence reduce noise pollution, travel time, property damage, fuel usages, and environmental pollution. Traditional traffic control systems do not possess the ability to manage with varying environments where many different factors can affect the decision-making processes. With the RFID tag system, it introduces a system that increases efficiency in routing traffic and overall reducing the amount of traffic in a specific area.

\section{SIGNIFICANCE OF THE STUDIES}

The Philippines is notorious for its heavy traffic congestion. Each and everyday traffic is present in almost every part of the major cities of the Philippines, especially in metro manila. People have a habit of breaking traffic rules, and there is also a lack of infrastructure. There was a study that even stated that in the Philippines, the yellow light did not make drivers slow down, but made them speed up [14]. There have also been proposals on an app to showcase the traffic all around metro manila [15], but what better way than to integrate the RFID system into this traffic management application. After years of development, the RFID has come to a point where automated systems or smart systems are made possible. Just like how some systems are used in warehouses to automate the production lines, or how they automate cars [16], this is also the goal for the traffic management system in the Philippines. With this, it will not only ease traffic, but it would also lead to a more efficient system that would help other factors such as the fuel economy. Under the current president's rule where the main goal is to build more roads and infrastructure, this system would pair well with it to bring the nation to a better state when it comes to traffic.

\section{DESCRIPTION OF THE SYSTEM}

The system consists of two main parts, the simulation of the RFID in the transmission end, and the simulation of the RFID in the receiving end. For the transmission simulation of the RFID, the source coding used in the simulation is the Manchester coding block which is a simple source encoding block that is easily received and is used due to AC coupling. The filter used after this is the raised cosine filter, a filter that is similar to a low pass Nyquist filter, giving the signal odd symmetry. There is also the use of the Hilbert transform, non-ideal mixers, high powered amplifiers and many more. It is then passed on to a digital to analog converter before it is sent across the simulated environment. An added feature is also a switch that can be used to manually shift from SSB to DSB and vice versa. The receiving end on the other hand comprises a simulated environment that makes of the AWGN channel which gives it the noise factor, a block that simulates path loss and a block that simulates the phase changes due to the distance that would be present between the tag and reader. This is then sent to the receiver which comprises blocks like the mixers, channel select filters, bandpass filters, and variable gain stages. This also contains the tag reflection part of the simulation. Figures 1 and 2 shows the Transmitter and Receiver MATLAB Simulation.

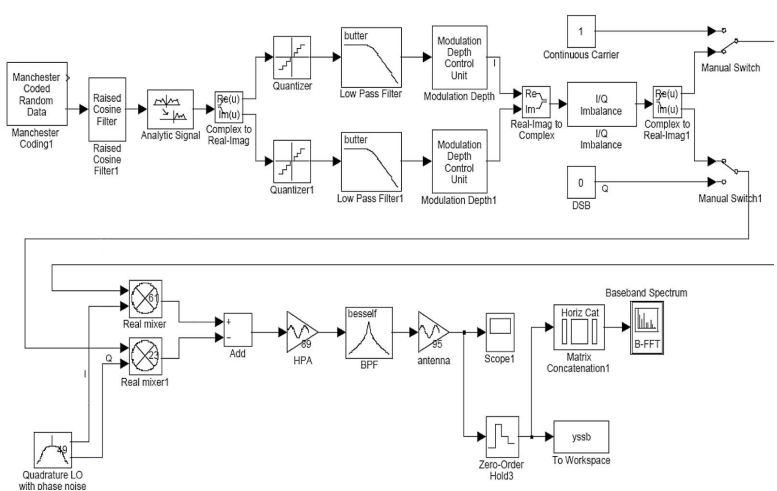

Figure 1:Transmitter Simulation in MATLAB-Simulink 


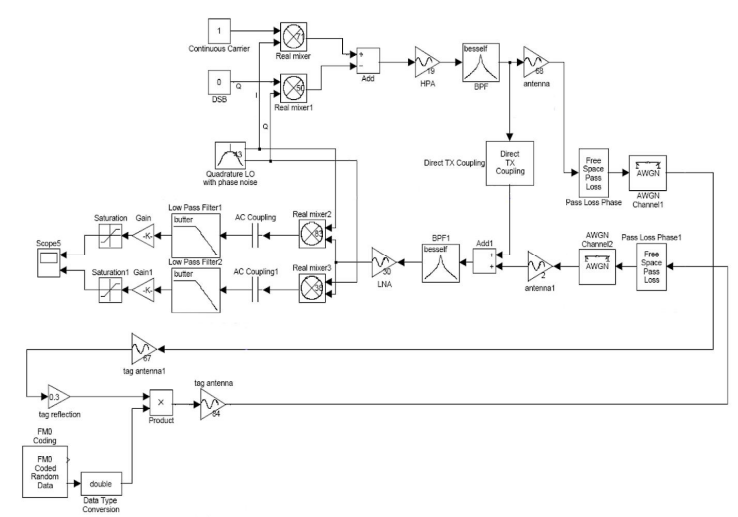

Figure 2: Receiver Simulation in MATLAB-Simulink

\section{METHODOLOGY}

Figures 3 and 4 shows the system flowchart.

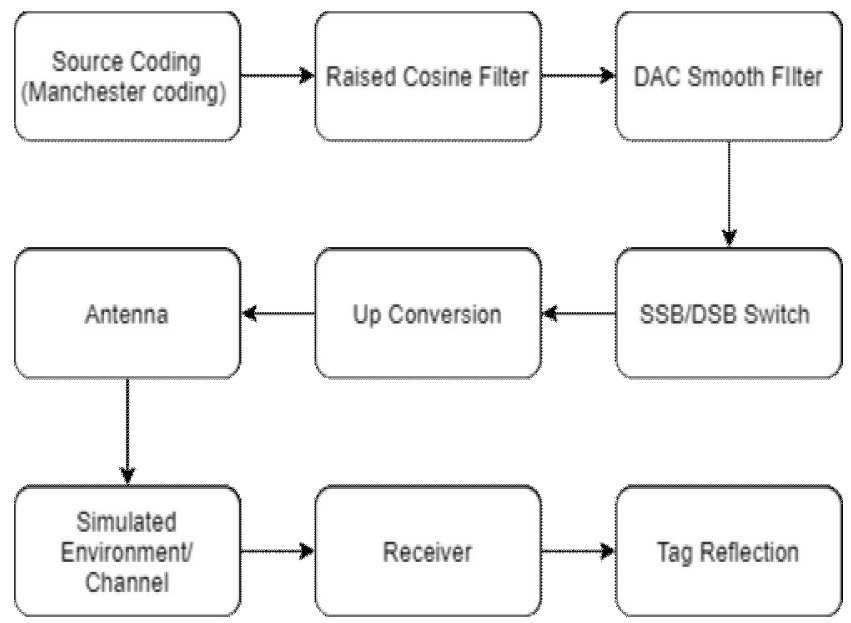

Figure 3:Simulation Flowchart

The RFID experiment is to be done in MATLAB using the computations of the antenna properties to determine what parameters are needed for an effective RFID tag reading in the road in a small scale trial. RFID is built as a transceiver so the transmitter of the signal must have fixed parameters, save for the frequency used, and the distance it has from the RFID tags. A vector network analyzer is to accompany the system using S-parameters to measure the effectiveness of the results in numbers. Once the set-up is done, the detection error rate for the tag that passes through the reader is analyzed. To acquire the DER, a high number of set data must be used when performing in MATLAB. To further make the results more realistic, added white Gaussian noise (AWGN) is added. With each trial, the frequency transmitted by the transmitter and the length of the RFID tag is varied. The results are to be observed after every transition, and the results will be recorded for comparison.

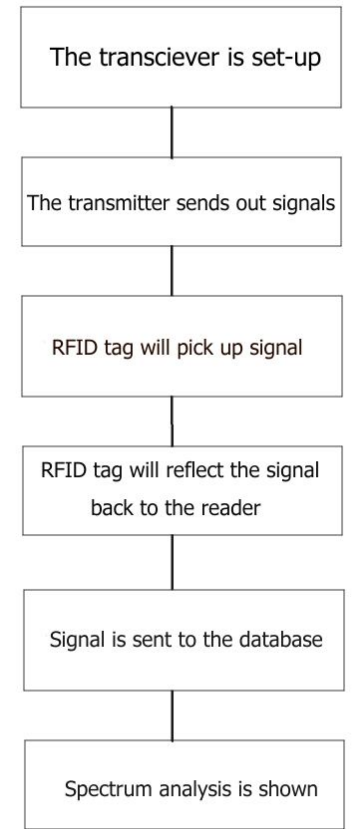

Figure 4:Experimentation Flowchart

\section{THEORETICAL CONSIDERATIONS}

There are several things to consider when simulating an RFID. The researcher must first understand the components to be used in making a simulated RFID reader and tag. This simulation made use of a simple simulation of a wireless RFID channel with a simple tag reflection. The source coding used in the simulation is the manchester coding block which is a simple source encoding block that is easily received. The filter used after this is the raised cosine filter, a filter that is similar to a low pass Nyquist filter, giving the signal odd symmetry [17]. It is then passed on to a digital to analog converter before it is sent across the simulated environment. To simulate the environment properly the AWGN channel was used to simulate noise that would be encountered in real life. All of these factors have been considered when making the simulation for it to be as near to real as possible while still keeping it simple and functional [18].

\section{DATA AND RESULTS}

Figures 5 to 9 shows the output of the simulations.

\subsection{DSB Transmitter Simulation at 80kbps}

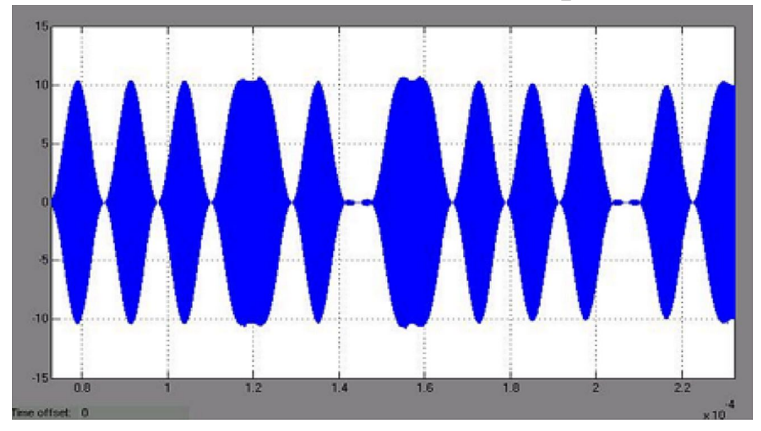

Figure 5: $100 \%$ modulation simulation at $80 \mathrm{kbps}$ 


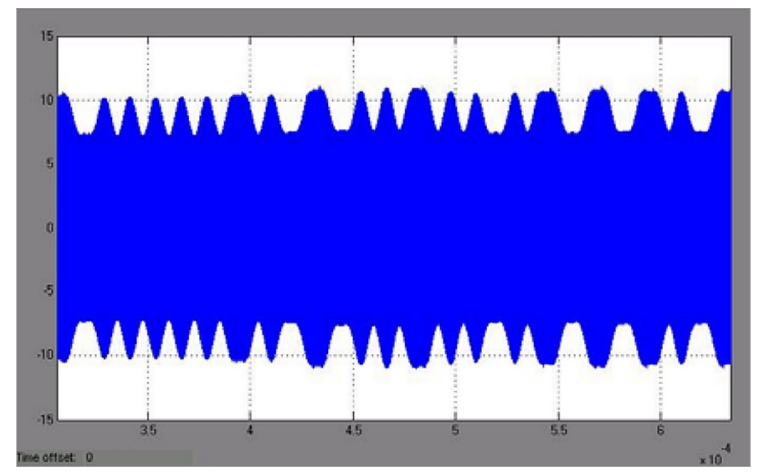

Figure 6:30\% modulation simulation at $80 \mathrm{kbps}$

\subsection{SSB Transmitter Simulation at 80kbps}

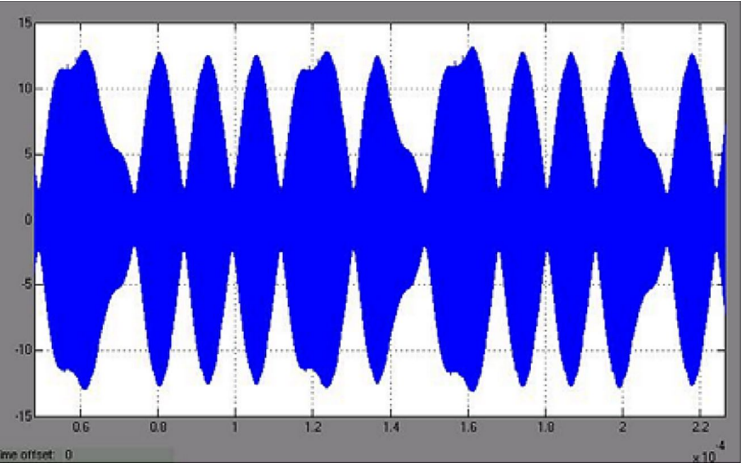

Figure 7:100\% modulation simulation at $80 \mathrm{kbps}$

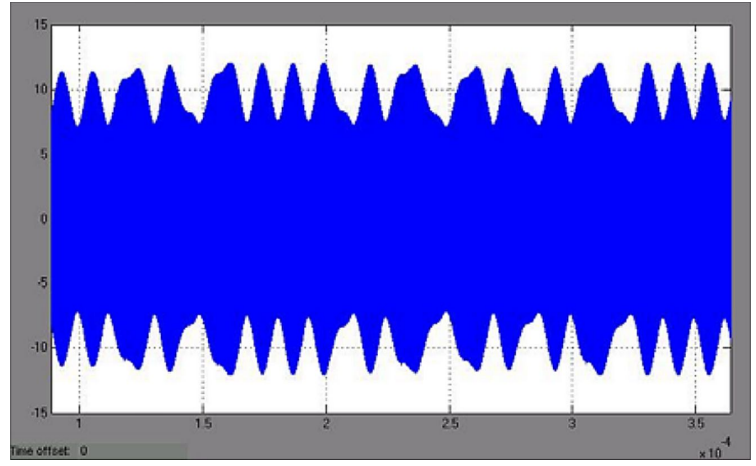

Figure 8: $30 \%$ modulation simulation at $80 \mathrm{kbps}$

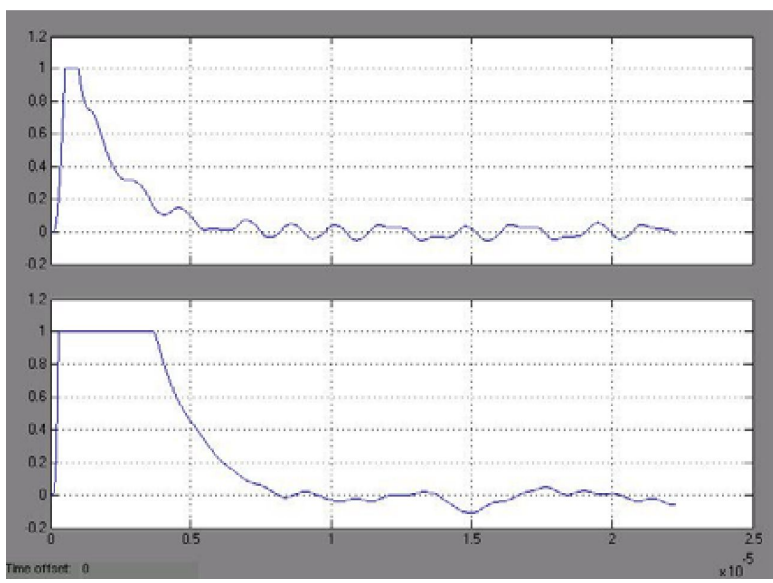

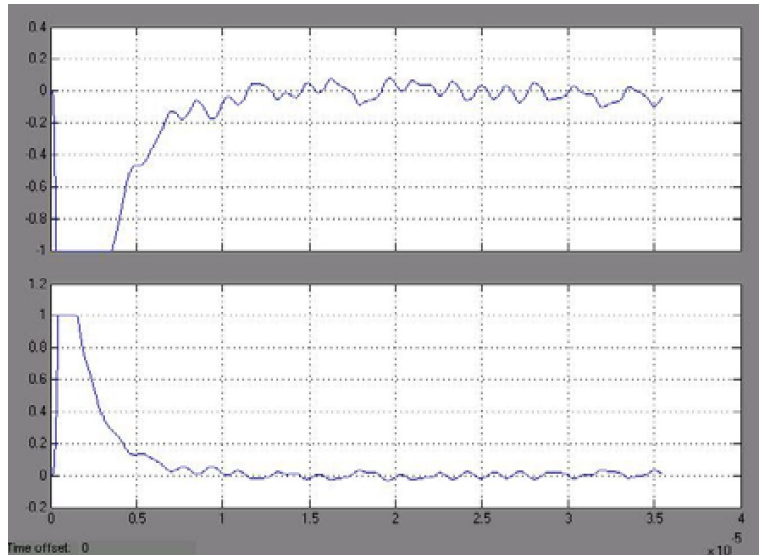

Figure 9: Signals retrieved by the receiver

\section{ANALYSIS OF DATA}

The results of the gathered data shown (Figure 9) through the transmitter and receiver signals with the return link is shown. With the graph shown, the signals are saturated because of the large DC offset. From the referred data and study, the signal-to-noise ratio is also better compared to the lower graph which means different results are determined by the phase delay changes of the direct coupling and the distance of the operation from where the tag is being read. The low-frequency noise and the fluctuation of amplitude in the graphs shown are almost permanent as it is more or a realistic simulation rather an ideal one. The crucial part of designing this kind of system of RFID reader is the circuit of the detector which will eventually show the supposed received data. The SSB transmission data gathered however is not a very good basis for simulation since, as mentioned earlier, is limited only to a finite length of data. With the detector, saturation is apparent whenever there is the suppression of the DC offset and transient response. One last critical part of the system is the bandwidth of the channels as it is related to noise floor.

\section{CONCLUSION}

This paper shows that the RFID system that was proposed at the start is possible to be simulated in MATLAB Simulink. The transmitting RFID tag and the receiving RFID reader was clearly and successfully simulated. Not only that but the real-life environment was also simulated using the communication toolbox from Simulink. The process of simulating showed that DSB or double sidebands were better suited for transmission when compared to the alternative SSB or single sidebands transmission. This happens as the SSB transmission is not optimized since a finite number of data is processed. During the testing phase, it was also deduced that the raised cosine filter needed to be looked after as it has a direct effect on the spectrum of the output. Not only that but as it was also noted on how the suppression of DC offset and transient response drove the circuit into saturation, this must also be taken into account when looking at the system as a whole. The simulated environment in the paper also focused its effect mainly on the RFID tag, and due to this, the 
bandwidth was also taken into account as it would dictate the amount of noise present in the system itself. Since this paper focused heavier on the RFID reader's end, there can be several more researches after this that could not only focus on the RFID tag but also on the simulation environment as a whole to make it even more realistic and complex. This paper did well to show a simple simulation of a wireless RFID channel with a simple reflection tag. It shows a comparison of the SSB and the DSB and it clearly simulated how the system would work in real life, utilizing several Simulink blocks and toolboxes. The application of the system could range anywhere from real-life traffic management applications, or to applications in the industrial and production lines. It shows that with proper simulation, one can clearly see the effects of real-life applications even when simply using software programs such as MATLAB.

\section{RECOMMENDATIONS}

Since this paper did well to show a simple simulation of a wireless RFID channel with a simple reflection tag, below are the blocks used. The source coding used in the simulation is the manchester coding block which is a simple source encoding block that is easily received. The filter used after this is the raised cosine filter, a filter that is similar to a low pass Nyquist filter, giving the signal odd symmetry. It is then passed on to a digital to analog converter before it is sent across the simulated environment. To simulate the environment properly the AWGN channel was used to simulate noise that would be encountered in real life. All of these factors have been considered when making the simulation for it to be as near to real as possible while still keeping it simple and functional. All of this is done with a focus on the transmission end of the RFID or the reader. In future papers, since this simulation was quite simple it is recommended that the researchers further push on the complexity of the simulation to even further simulate what it would be like in a practical and real-life scenario. To do this, one can either add more blocks to Simulink or to even further utilize the capacity of MATLAB and use it to its full potential as the paper did not touch on other toolboxes other than the communications toolbox. Not only this but also that future research be conducted in the frequency domain to further simulate the reader and tag system of the RFID in a more complex and accurate manner. All of this is recommended to solve the problems faced in this paper and to further address the need for complex simulations that would satisfy the various applications this system can be implemented in.

\section{REFERENCES}

[1] D. M. Dobkin, "History and Practice of RFID," in The RF in RFID, 2nd ed, Newnes, pp. 7-47, 2013.

[2] D. M. Dobkin, "Introduction," in The RF in RFID, 2nd ed, Newnes, pp. 1-5, 2013.

[3] D. M. Dobkin, "Radio Basics for UHF RFID," in The RF in RFID, 2nd ed, Newnes, pp. 49-101, 2013.

[4] D. M. Dobkin, "UHF RFID Tags," in The RF in RFID, 2nd ed, Newnes, pp. 189-237, 2013.
[5] D. M. Dobkin, "UHF RFID Readers," in The RF in RFID, 2nd ed, Newnes, pp. 103-188, 2013.

[6] D. M. Dobkin, "Reader Antennas," in The RF in RFID, 2nd ed, Newnes, pp. 239-297, 2013.

[7] D. M. Dobkin, "Tag Antennas," in The RF in RFID, 2nd ed, Newnes, pp. 299-359, 2013.

[8] A. Bensky, "Radio propagation," in Short-range Wireless Communication, 3rd ed, Newnes, pp. 11-41, 2019.

[9] G. R. Filho, R. Meneguette, et al, "Enhancing intelligence in traffic management systems to aid in vehicle traffic congestion problems in smart cities," Ad Hoc Networks, Vol. 107, 2020.

[10] I. El Masri, B. Lescop, et al, "Development of a RFID sensitive tag dedicated to the monitoring of the environmental corrosiveness for indoor applications," Sensors and Actuators B: Chemical, Vol. 322, 2020.

[11] W. M. Abdulkawi, A. A. Sheta, "Chipless RFID Sensors Based on Multistate Coupled Line Resonators, Sensors and Actuators," Sensors and Actuators A: Physical, Vol. 309, 2020.

[12] M. Pekgor, M. Nikzad, et al, "Sensor-based filament fabrication with embedded RFID microchips for 3D printing," Materials Today: Proceedings, 2020.

[13] N. J. Kohut, J. K. Hedrick, et al, "Integrating Traffic Data and Model Predictive Control to Improve Fuel Economy," Vol. 42, no. 15, pp.155-160, 2009.

[14] G. P. Felicio, L. C. Grepo, et al, "Traffic Light Displays and Driver Behaviors: A Case Study," Procedia Manufacturing, Vol. 3, pp. 3266-3273, 2015.

[15] Emmanuel Yujuico, "Considerations in the diffusion of a public traffic app for Metro Manila," Journal of Transport Geography, Vol. 42, pp. 48-56, 2015.

[16] S. Lu, C. Xu, et al, "A passive RFID tag-based locating and navigating approach for automated guided vehicle," Computers \& Industrial Engineering, Vol. 125, pp. 628 636, 2018

[17] A. Africa, S. Wu, I. Naco, J. Castillo and V. Valdes, "Modes of radio wave propagation: Troposcatter," International Journal of Emerging Trends in Engineering Research, Vol. 8, No. 4, pp. 1175-1179, 2020.

[18] A. Fadl, M. Ihedrane, and S. Bri, "Estimation direction arrival of smart antenna," International Journal of Emerging Trends in Engineering Research, Vol. 8, No. 4, pp. 1051-1055, 2020. 